\title{
Pedestrians' needs in the urban environment: the case of the city of Trikala, Greece
}

\author{
S. Basbas, C. Konstantinidou \& N. Gogou \\ Faculty of Rural and Surveying Engineering, \\ Aristotle University of Thessaloniki, Greece
}

\begin{abstract}
Walking has become one of the most important transport modes in urban areas. The role of walking has steadily increased worldwide and therefore city planners put as their first priority the facilitation of pedestrian trips in urban areas. In doing so, several aspects pertaining to pedestrians trips need to be addressed, such as safety, environment, accessibility, security, etc. This paper attempts to address the needs of pedestrians in the city of Trikala, Greece. In the framework of this research a questionnaire-based survey was carried out in the centre of the city of Trikala. Special emphasis is given to different age groups because the elderly usually face more difficulties during their trips made on foot compared to other age groups. In addition, the survey provides information about the reasons for choosing walking as a means of transport, the difficulties people face during their trips, the level of safety as perceived by the pedestrians, etc. Moreover, people's suggestions about possible methods of making the walking environment friendlier are also recorded. It is believed that the results of the specific research will help city planners in their effort to improve the walking environment and promote walking as a sustainable method of transport.
\end{abstract}

Keywords: walking, pedestrians, transport.

\section{Introduction}

One of the most important needs of pedestrians is the need for comfortable and safe mobility. This need is even more essential for vulnerable road users. "Vulnerable road users" is a term commonly used to describe those groups who are likely to suffer when in conflict with cars, buses and other large vehicles. It 
may also be used to describe users of the highway whose mobility is in some way reduced or who face barriers when they move in the highway environment.

These road users include pedestrians, elderly people, children, people with small children, people with mobility disabilities, people whose mobility is impaired due to pregnancy or because they are carrying any type of heavy luggage [1]. In particular for the majority of older people, walking is vital to their mobility, particularly for short trips and carrying out essential daily tasks. Moreover, there are noted environmental and health benefits associated with walking for all aged adults, but particularly for older adults [2].

This paper attempts to address the main needs of pedestrians and the difficulties that they meet during their trips in terms of safety and accessibility. The identification of needs is based on a survey that was carried out in the city of Trikala, Greece. Additionally, the reasons for which someone takes the decision to walk together with the identification of measures in order to improve the walking environment are also included in the paper.

\section{Pedestrian needs}

According to COST 358: Pedestrian Quality Needs, there are four basic needs of pedestrians; "the need for basic mobility", "the need for safe mobility", "the need for convivial mobility" and "Challenging Sojourn" [3].

Pedestrian needs can be looked upon in different ways. COST 358: Pedestrian Quality Needs identify "functional" and "perceived" needs of pedestrians. Functional needs are the pedestrians' physical needs. One way to identify functional needs is through observation and surveys. Pedestrian behaviour reveals their functional needs. Obviously, functional needs differentiate among different pedestrian groups. Figure 1 shows a Maslow-like hierarchy of functional needs, which are divided into 3 levels: preconditions, dissatisfyers and satisfyers.

On the other hand, perceived needs are the pedestrian needs which are related with the emotional point of view. They are determined by studying the perceptions of pedestrians or potential pedestrians.

People's perceptions are affected by many factors such as the social norms, education, the environment, experiences etc. "As society changes many of these factors change and consequently perceptions about pedestrian needs change too. One major issue when examining perceived needs of pedestrians is the question what are the required facilities and provisions for the pedestrians in order to perform pedestrian tasks adequately" [4].

According to Townsend [5] the factors which make walking environment adequate for pedestrians are: smooth footpaths, wide footpaths, adequate connection streets, safe crossings, good shortcuts, seating, adequate lighting, few cars, low speeds, clean air, footpaths with trees, friendly buildings. The present paper attempts to address the needs of pedestrians in the city of Trikala, Greece. In the framework of this research a questionnaire-based survey was carried out in the centre of the city of Trikala. 


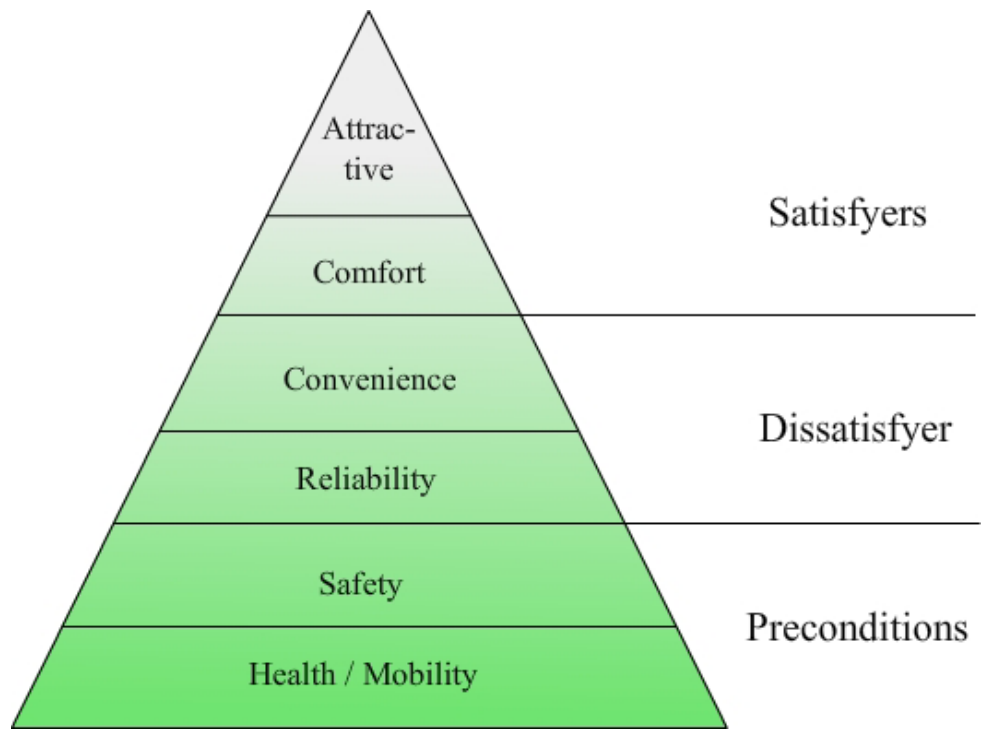

Figure 1: $\quad$ Maslow-like hierarchy of pedestrian functional needs (source [3]).

The city of Trikala has a population of 48.686 residents according to the census of the year 2001 [6] and faces traffic problems especially during peak periods. According to a public transport study which was carried out in year 2000 [7] 31,4\% of the daily trips were pedestrian trips, 10,7\% were made by bicycles, $10,3 \%$ by motorcycles, $32,6 \%$ by private cars, $10,5 \%$ by public transport buses etc.

A questionnaire based survey in the centre of the city of Trikala [2], as mentioned above, was carried out during the end of April and May 2008 where 150 questionnaires were used for the specific research which is presented in this paper.

\section{Difficulties experienced by the pedestrians and reasons for choosing walking}

The road networks were originally designed in order to facilitate vehicular traffic and little attention was paid to pedestrians and cyclists. For this reason, difficulties during pedestrian trips are expected. Nowadays special attempts have been made by engineers, designers and transport planners in order to reduce pedestrians' barriers and improve the walking environment in order to attract new users.

There are many difficulties which the pedestrians in the city of Trikala have referred to. Some of these are presented in the following Figure 2. The absence of green areas $(20 \%)$ and degraded environment (19\%) are the main barriers to pedestrians' trips while a percentage of 5\% feel uncomfortable during walking 


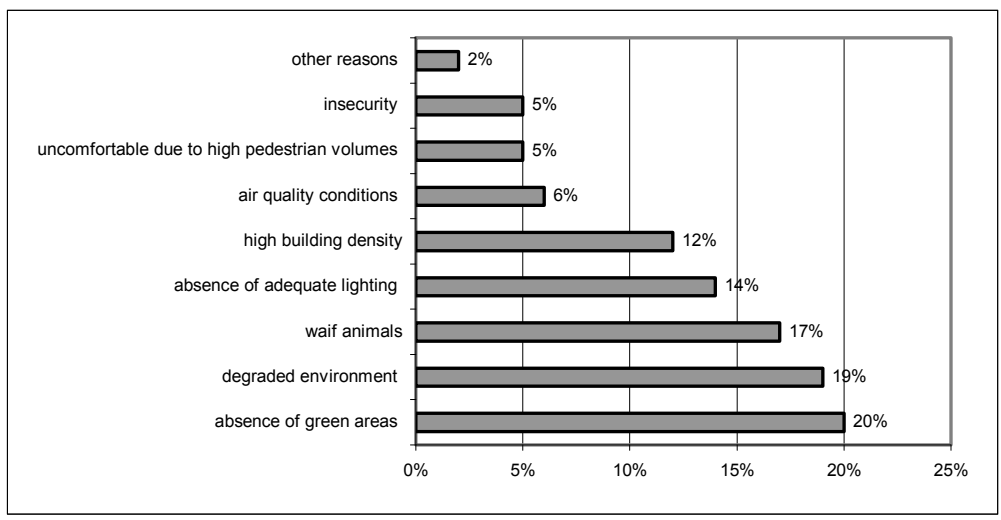

Figure 2: Difficulties during pedestrians’ trips.

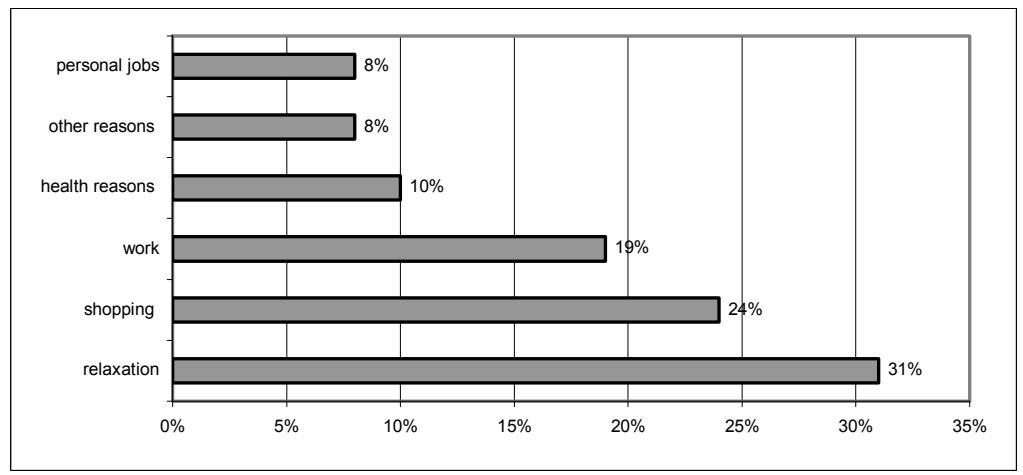

Figure 3: $\quad$ Reasons for walking.

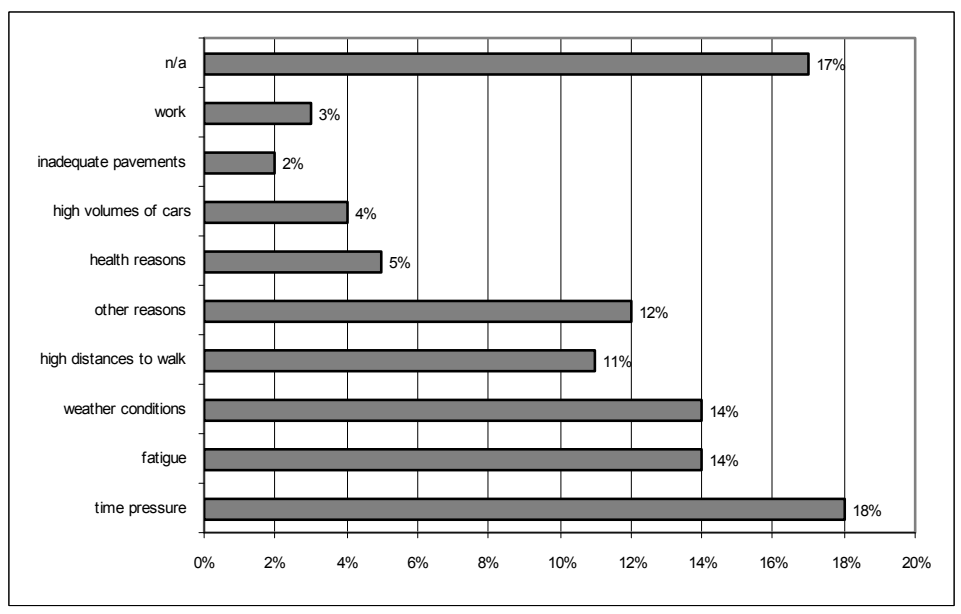

Figure 4: Reasons for not walking. 
due to high pedestrian volumes and insecurity. Concerning the reasons for walking, Figure 3 presents these.

As it is presented in Figure 3, 31\% of the sample mentioned that they walk for relaxation and $24 \%$ for shopping while a percentage of $8 \%$ mentioned that they walk for other reasons (i.e. exercise) or personal jobs. Reasons that deter people from walking are presented in Figure 4. Time pressure $(18 \%)$ is the main reason for not walking while an inadequate pavement $(2 \%)$ is the last reason which prevents people from walking.

\section{Feeling of safety and feeling of comfort}

The feeling of safety during walking is one of the most important factors which play a crucial role during people's decision for walking. According to the results from an American telephone survey, which was carried out in October 2002 by Belden Russonello and Stewart [8], (national random sample of 800 adults, age 18 and older from October 23 to 30, 2002) Americans would like to walk more but speeding and inadequate infrastructure such as dangerous intersections discouraged them.

Americans support policies to make walking safer and easier. $86 \%$ of Americans desire better enforcement of traffic laws, such as speed limits $(57 \%$ strongly favour) [8]. Over eight in ten (84\%) desire more money for the transportation sector in order to design streets with sidewalks, safe crossing and other devices to reduce speeding in residential areas and make it safer to walk, which means driving more slowly (48\% strongly) [8].

The research results about the feeling of safety in the city of Trikala are presented in the following Figures 5 and 6.

Pedestrians on the pavements in the city of Trikala feel safe $(55 \%)$ while a percentage of $45 \%$ feel unsafe during their trips on foot (Figure 5). In terms of age group, the older people feel more unsafe during their movements on pavements $(56 \%)$ than other age groups who can walk more easily than the elderly. This means that special attention should be paid to vulnerable road users and especially to the elderly. In terms of pedestrians' opinions about the walking environment, the majority of them did not find it comfortable and friendly for their trips, as it can be noticed in Figures 7 and 8 .

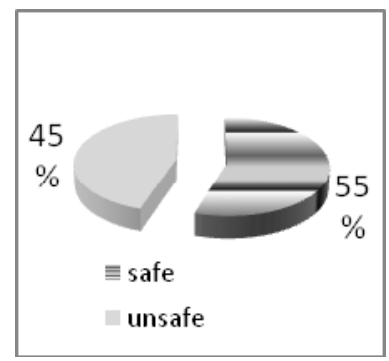

Figure 5: $\quad$ Feeling of safety. 


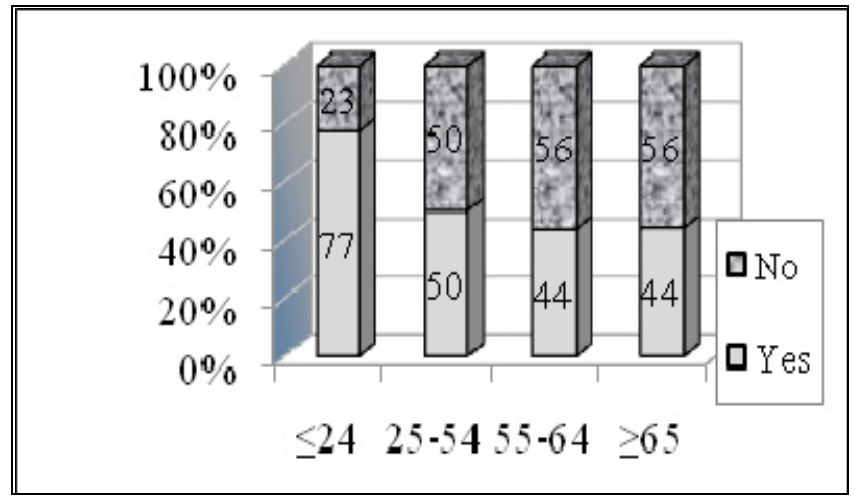

Figure 6: $\quad$ Age group and feeling of safety.

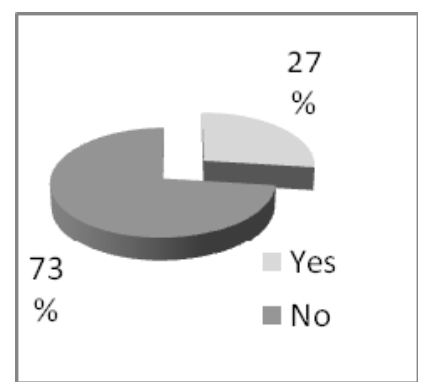

Figure 7: Friendly walking environment.

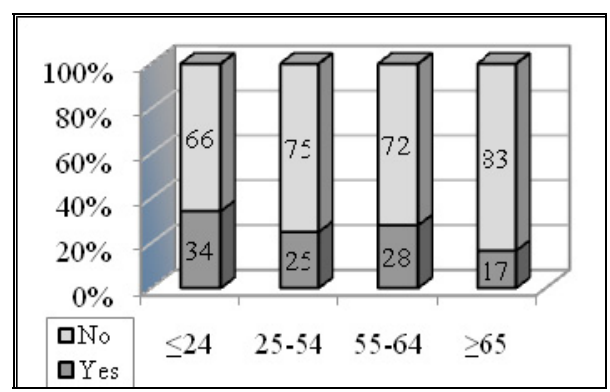

Figure 8: Age groups' opinion.

A percentage of $73 \%$ of pedestrians mentioned that walking environment in the city of Trikala is not friendly and their trips are not comfortable while $27 \%$ of the sample mentioned that the walking environment is comfortable enough and friendly. According to the opinion of different age groups about the walking environment, the elderly find walking environment less friendlier and comfortable than the young with the people who are $\geq 65$ years old to mention that walking environment is not accessible at a percentage of $83 \%$. 


\section{Measures proposed by the pedestrians}

According to the survey results, pedestrians propose different measures so that walking environment becomes friendlier. Figure 9 presents these proposed measures.

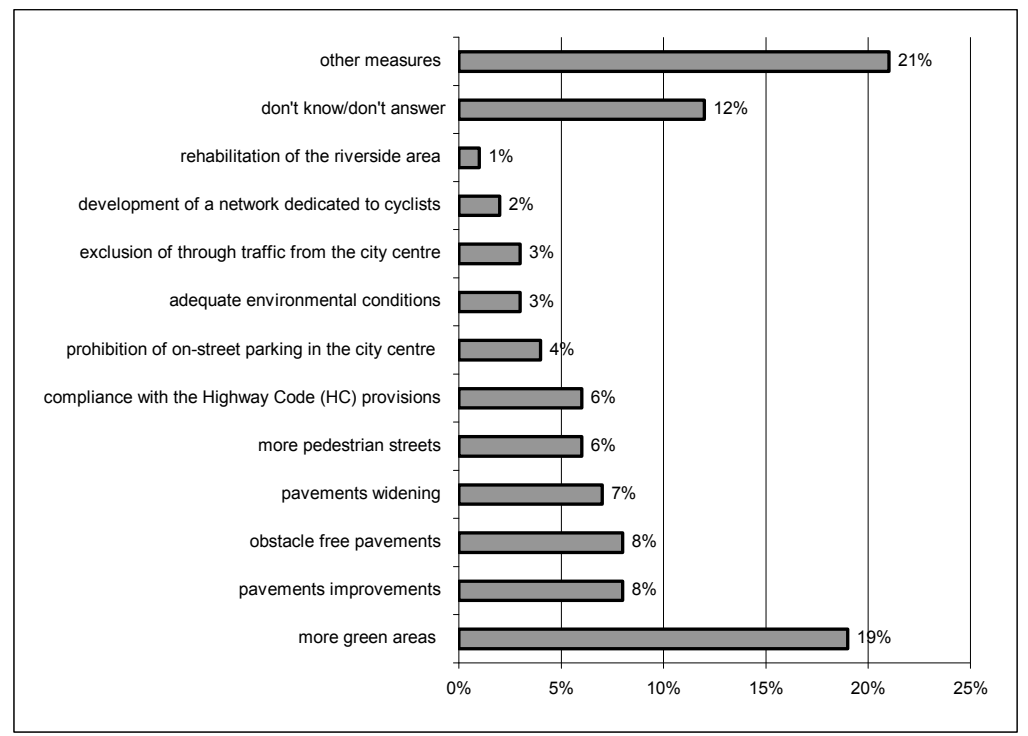

Figure 9: Proposals for making walking more attractive.

As it can be noticed from the above Figure 9, a percentage of $19 \%$ of the people require more green areas. Other measures refer to improvements of pavements, extension of pedestrian streets, Highway Code provisions for pedestrians, motorized traffic restraint measures etc.

\section{Discussion}

The difficulties which pedestrians experience during their trips make walking environment more and more unfriendly in many cases. As a result, people prefer to use private or public means of transport for their trips rather than to walk in case where walking would be an option too. The problem becomes bigger in the case of vulnerable road users and especially for the elderly and people with special mobility needs.

Transport planners should take into consideration a series of measures in order to improve walking environment such as adequate speed limits, traffic calming measures, pedestrianisation schemes, ramps and other infrastructure for people with special mobility needs, use of Intelligent Transport Systems (ITS) like Variable Message Signs (VMS), CCTV systems etc. 
The local authorities should make walking environment (aesthetics, air quality, greener areas) safe and secure for people. Only then infrastructure dedicated to pedestrians will attract new users. In the case of the city of Trikala, it seems that pedestrians are not satisfied with the offered walking environment. They required better conditions and facilities so that their trips on foot can be made friendlier, especially for vulnerable road users. It is believed that the results of the specific research will help city planners in their effort to improve walking environment and promote walking as a sustainable way of transport.

\section{References}

[1] Apostolidis, Ch, Gaitazi, X. \& Kitsou, E. Evaluation of pedestrians characteristics in the centre of Thessaloniki, Diploma Thesis, Supervisor: Basbas, S., Faculty of Rural \& Surveying Engineering, Aristotle University of Thessaloniki, 2008.

[2] Gogou, N. Evaluation of pedestrians characteristics in the centre of Trikala, M.Sc Thesis, Supervisor: Basbas, S., Faculty of Rural \& Surveying Engineering, Aristotle University of Thessaloniki, 2009.

[3] Methorst, R. Assessing Pedestrians' Needs, The European COST 358 PQN project. Proc. Of the $8^{\text {th }}$ Annual International Conference on Walking and Liveable Communities, Walk 21, Putting Pedestrians First, Toronto, Canada, pp.1-21, 2007.

[4] Basbas, S., Konstantinidou, C., Papaioannou, P. \& Politis, I. Pedestrian Functional and Perceived Needs in the Urban Environment. Proc. of the International Conference on Accessibility and Safety for All Thessaloniki, 2009.

[5] Townsend, L. What do people want in the walking environment? IUM partner, $15 \mathrm{p}$.

[6] National Statistical Service of Greece (NSSG), www.statistics.gr

[7] Panourgias, X., Gougoulias, D. Study for the development of Municipal Public Transport System in the city of Trikala, Municipality of Trikala, October 2000.

[8] Belden Russonello \& Stewart, Research and Communications, Americans' Attitudes toward Walking and Creating Better Walking Communities., Washington, D.C., April 2003. 